Portland State University

PDXScholar

\title{
Column-grid-array (CGA) versus Ball-grid-array (BGA): Boardlevel Drop Test and the Expected Dynamic Stress in the Solder Material
}

\author{
Ephraim Suhir \\ Portland State University \\ Reza Ghaffarian \\ Jet Propulsion Laboratory
}

Follow this and additional works at: https://pdxscholar.library.pdx.edu/mengin_fac

Part of the Materials Science and Engineering Commons

Let us know how access to this document benefits you.

\section{Citation Details}

Suhir, E., \& Ghaffarian, R. (2016). Column-grid-array (CGA) versus ball-grid-array (BGA): board-level drop test and the expected dynamic stress in the solder material. Journal of Materials Science: Materials in Electronics, 27(11), 11572-11582.

This Article is brought to you for free and open access. It has been accepted for inclusion in Mechanical and Materials Engineering Faculty Publications and Presentations by an authorized administrator of PDXScholar. Please contact us if we can make this document more accessible: pdxscholar@pdx.edu. 


\title{
Column-grid-array (CGA) versus ball-grid-array (BGA): board- level drop test and the expected dynamic stress in the solder material
}

\author{
E. Suhir ${ }^{1,2} \cdot$ R. Ghaffarian ${ }^{3}$
}

Received: 29 March 2016/Accepted: 29 June 2016/Published online: 12 July 2016

(C) Springer Science+Business Media New York 2016

\begin{abstract}
Board level drop test is considered with an objective to develop a physically meaningful analytical predictive model for the evaluation of the expected impactinduced dynamic stresses in the solder material. Ball-gridarray (BGA) and column-grid-array (CGA) designs are addressed. Intuitively it is felt that while the application of the CGA technology to relieve thermal stresses in the solder material might be quite effective (owing to the greater interfacial compliance of the CGA in comparison with the BGA), the situation might be quite different when the $\mathrm{PCB} /$ package experiences dynamic loading. This is because the mass of the CGA joints exceeds considerably that of the BGA interconnections and the corresponding inertia forces might be substantially larger in the case of a CGA design. The numerical example carried out for rather arbitrary, but realistic, input data has indicated that the dynamic stresses in the solder material of the CGA design are even higher than the stresses in the BGA interconnections. This means particularly that the physically meaningful drop height in board-level tests should be thoroughly selected and that this height should be different, for BGA and CGA designs.
\end{abstract}

E. Suhir

suhire@aol.com;

http://www.ERSuhir.com

R. Ghaffarian

reza.ghaffarian@jpl.nasa.gov

1 Portland State University, Portland, OR, USA

2 ERS Co., 727 Alvina Ct., Los Altos, CA 94024, USA

3 Jet Propulsion Laboratory, California Institute of Technology, Pasadena, CA, USA

\section{Introduction}

It has been established [1-10] that compliant attachments can provide substantial buffering effect, thereby leading to an appreciable relief in thermally induced stresses in adhesively bonded or soldered assemblies, including, first of all, the attachment itself. It has been shown also [11] that a similar effect takes place in the case of mechanical loading, when a bi-component assembly experiences the combined action of tensile forces and bending moments applied to the ends of one of its components (Fig. 1).

In the study that follows these forces and moments are determined from the analysis of the nonlinear dynamic response of the PCB to the drop impact during board level drop test. Its objective is to develop a simple and physically meaningful predictive analytical model that would enable one to evaluate the interfacial dynamic stresses in the solder material of the CGA and BGA designs. The analysis is an extension and a modification of the study [11].

\section{Analysis}

\subsection{Assumptions}

- Methods of engineering structural analysis are applicable (see e.g., $[12,13]$ ), as well as the concept of the interfacial compliance that was initially applied to the case of thermal stresses [1-3]; this concept separates the roles of the materials-and-structural characteristics and the role of the external loading; this concept is applicable regardless of the nature of the applied load;

- The longitudinal cross-section of the assembly can be considered, instead of addressing the actual two- or 


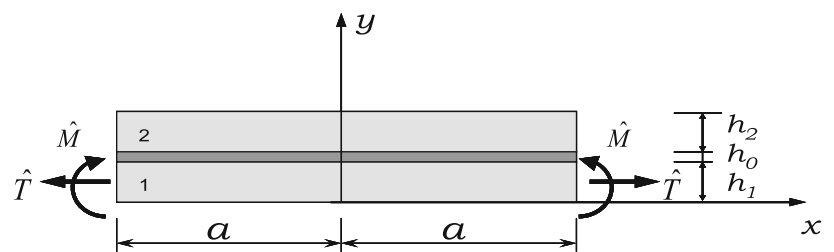

Fig. 1 Bi-material assembly subjected to tensile forces and bending moments applied to one of its components: the lower component \#1 (PCB) experiences direct action of the external tensile forces and bending moments, while the upper component \#2 (package) is loaded by the interfacial shearing and peeling forces transmitted through the bonding layer

even three-dimensional structure, as it has been done in several previous publications (see e.g., [1-3]);

- A continuous (homogeneous) layer of the solder material can be considered instead of the actual (inhomogeneous) solder system, with small gaps between the solder joints [14];

- The tensile forces $\hat{T}$ and bending moments $\hat{M}$ (per unite package width) applied in the package/PCB assembly to the edges of the $\mathrm{PCB}$ can be determined for packages, whose size is assumed to be considerably smaller than the PCB size and in a conservative analysis, by the formulas [15]

$\hat{T}=\frac{E}{1-v} \frac{2-v}{2} h\left(\frac{\pi A}{4 l}\right)^{2}, \quad \hat{M}=\frac{E}{1-v} \frac{h^{3}}{12} A\left(\frac{\pi}{2 l}\right)^{2}$

Here $E$ is the effective Young's modulus of the PCB material, $v$ is its Poisson's ratio, $h$ is the board's thickness, $A$ is the amplitude of (nonlinear) vibrations, and $l$ is half of the PCB length. When the forces $\hat{T}$ and bending moments $\hat{M}$ are obtained from the measured strains in an actual experiment (Figs. 2, 3), they can be evaluated by the formulas:

$\hat{T}=\frac{E h}{1-v} \frac{\varepsilon_{1}+\varepsilon_{2}}{2}, \quad \hat{M}=\frac{E h^{2}}{6\left(1-v^{2}\right)} \frac{\varepsilon_{1}-\varepsilon_{2}}{2}$,

where $\varepsilon_{1}$ and $\varepsilon_{2}$ are the strains measured on the upper and the lower surfaces of the PCB in the proximity of the assembly of interest.
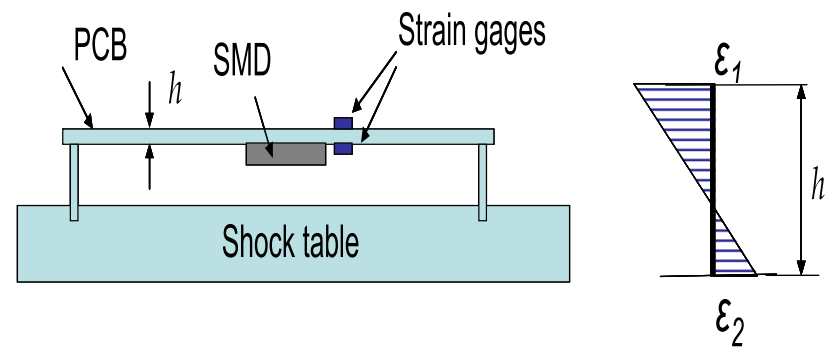

Fig. $2 \mathrm{PCB} /$ package assembly with strain gages mounted on a shock table

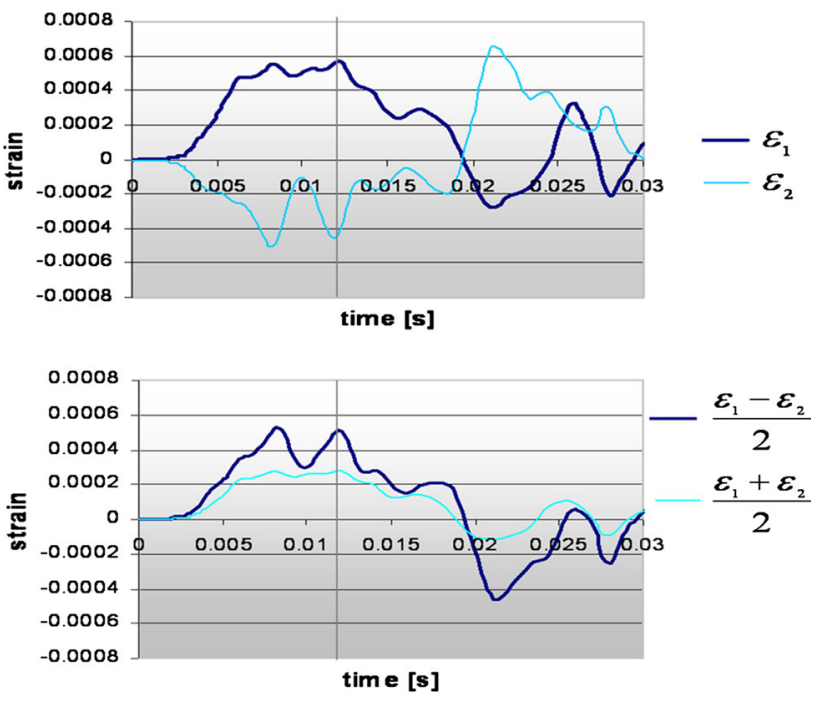

Fig. 3 Typical strain distribution obtained from strain readings at the instant of peak board deflections; strain recording (top), computed bending and membrane component of the strain (bottom). The forces and the moments are computed at the time $\mathrm{t}=0.012 \mathrm{~s}$ (gray line on strain plots). The measurements and calculations were carried out by Dr. Vujosevic, Intel

\subsection{Longitudinal interfacial displacements}

Let a bi-material PCB/package assembly be subjected to tensile forces and bending moments applied to one of its components (Fig. 1). Using the concept of interfacial compliance [1], the longitudinal interfacial displacements of the assembly components experiencing external (mechanical) loading can be sought in the form:

$$
\begin{gathered}
u_{1}(x)=\lambda_{1} \int_{0}^{x} T_{1}(\xi) d \xi+\kappa_{1} \tau(x)-\frac{h_{1}}{2} w_{1}^{\prime}(x), \\
u_{2}(x)=\lambda_{2} \int_{0}^{x} T_{2}(\xi) d \xi-\kappa_{2} \tau(x)+\frac{h_{2}}{2} w_{2}^{\prime}(x) .
\end{gathered}
$$

Here $u_{1}(x)$ is the interfacial displacement of the component \#1 (PCB), $u_{2}(x)$ is the interfacial displacement of the component \#2 (package),

$\lambda_{1}=\frac{1-v_{1}}{E_{1} h_{1}}, \quad \lambda_{2}=\frac{1-v_{2}}{E_{2} h_{2}}$

are the longitudinal axial compliances of these components, $E_{1}$ and $E_{2}$ are Young's moduli of their materials, $v_{1}$ and $v_{2}$ are their Poisson's ratios; $h_{1}$ and $h_{2}$ are the components' thicknesses,

$\kappa_{1}=\frac{h_{1}}{3 G_{1}}, \quad \kappa_{2}=\frac{h_{2}}{3 G_{2}}$

are the longitudinal interfacial compliances of the components, 
$G_{1}=\frac{E_{1}}{2\left(1+v_{1}\right)}, \quad G_{2}=\frac{E_{2}}{2\left(1+v_{2}\right)}$

are the shear moduli of the materials, $\tau(x)$ is the interfacial shearing stress,

$T_{1}(x)=\hat{T}-T_{2}(x), \quad T_{2}(x)=\int_{-a}^{x} \tau(\xi) d \xi$

are the tensile forces acting in the cross-sections of the components $\# 1$ and $\# 2$, respectively, $\hat{T}$ are the external tensile forces applied to the ends of the component \#1 and expressed by the first formulas in (1) or (2), $a$ is half the $\mathrm{PCB} /$ package assembly length, and $w_{1}(x)$ and $w_{2}(x)$ are the deflection functions of the PCB and the package. The origin $\mathrm{O}$ of the longitudinal coordinate $x$ is in the midcross-section of the assembly.

The first terms in the formulas (3) are the axial displacements caused by the forces $T_{1}(x)$ and $T_{2}(x)$ and evaluated in accordance with Hooke's law, assuming that they are the same for all the points of the given crosssection. The second terms are corrections to this assumption. These corrections consider that the interfacial displacements are somewhat larger than the displacements of the inner points of the cross-section, i.e., the points sufficiently remote from the interface. The structure of the corrections reflects an assumption that the deviation from the cross-section's planarity at the assembly interface can be evaluated as a product of the induced interfacial shearing stress, $\tau(x)$, acting in this cross-section, and the pre-determined (loading independent) interfacial compliance, $\kappa$ of the given component. The formulas (5) were obtained based on the theory-of-elasticity solution for a long-and-narrow strip loaded over one of its long edges [1]. Since the compliance $\kappa$ is loading independent, the results obtained for the case of thermal loading are applicable to the mechanical loading in as well. It is assumed also that the above deviation from planarity is due only to the state of stress and strain in the given cross-section and is not affected by the states of stress and strain in the adjacent cross-sections. The third terms in the formulas (3) are the interfacial displacements caused by bending. These displacements are proportional to the angles of rotation of the components' cross-sections and are different, of course, for the convex and the concave sides of the component.

\subsection{Condition of displacement compatibility}

The condition of the compatibility of the displacements (3) can be written as

$u_{1}(x)=u_{2}(x)-\kappa_{0} \tau(x)$,

where
$\kappa_{0}=\frac{h_{0}}{G_{0}}$

is the interfacial compliance of the bonding layer, $h_{0}$ is the thickness of this layer,

$G_{0}=\frac{E_{0}}{2\left(1+v_{0}\right)}$

is the shear modulus of the bonding material, and $E_{0}$ and $v_{0}$ are the elastic constants of the bonding material.

\subsection{Basic equation for the interfacial shearing stress}

Introducing the formulas (3) for the displacements into the compatibility condition (7), the following equation for the sought shearing stress function, $\tau(x)$, can be obtained:

$$
\begin{aligned}
\kappa \tau(x)- & \left(\lambda_{1}\right. \\
& \left.+\lambda_{2}\right) \int_{0}^{x} T_{2}(\xi) d \xi-\frac{h_{1}}{2} w_{1}^{\prime}(x)-\frac{h_{2}}{2} w_{2}^{\prime}(x)=-\lambda_{1} \hat{T} x .
\end{aligned}
$$

Here

$\kappa=\kappa_{0}+\kappa_{1}+\kappa_{2}$

is the total interfacial compliance of the assembly. Unlike the axial compliances (4), which (in a situation, when the bond is characterized by the significantly higher axial compliance than the PCB and the package) are independent of the compliance of the bond, the interfacial compliance (11) of the assembly is affected by all the three constituent materials.

From (10) we obtain, by differentiation:

$\kappa \tau^{\prime}(x)-\left(\lambda_{1}+\lambda_{2}\right) T_{2}(x)-\frac{h_{1}}{2} w_{1}^{\prime \prime}(x)-\frac{h_{2}}{2} w_{2}^{\prime \prime}(x)=-\lambda_{1} \hat{T}$.

The next differentiation yields:

$\kappa \tau^{\prime \prime}(x)-\left(\lambda_{1}+\lambda_{2}\right) \tau(x)-\frac{h_{1}}{2} w_{1}^{\prime \prime \prime}(x)-\frac{h_{2}}{2} w_{2}^{\prime \prime \prime}(x)=0$.

\subsection{Basic equation for the peeling stress}

The equations of bending (equilibrium) of the assembly components (treated here as thin elongated plates) can be written as

$$
\begin{gathered}
D_{1} w_{1}^{\prime \prime}(x)=-\hat{M}+\int_{-a}^{x} \int_{-a}^{x} p(\xi) d \xi d \xi-\frac{h_{1}}{2} T_{1}(x), \\
D_{2} w_{2}^{\prime \prime}(x)=-\int_{-a}^{x} \int_{-a}^{x} p(\xi) d \xi d \xi+\frac{h_{2}}{2} T_{2}(x),
\end{gathered}
$$


where

$D_{1}=\frac{E_{1} h_{1}^{3}}{12\left(1-v_{1}^{2}\right)}, \quad D_{2}=\frac{E_{2} h_{2}^{3}}{12\left(1-v_{2}^{2}\right)}$

are the flexural rigidities of the assembly components, $\hat{M}$ are the external bending moments (per unit assembly width) acting at the ends of the component \#1 and expressed by the second formulas in (1) and (2), $p(x)$ is the interfacial peeling stress (i.e., the interfacial normal stress acting in the through-thickness direction of the assembly), and the double integrals express the bending moments caused by the loading $p(x)$.

We assume that the peeling stress, $p(x)$, is proportional to the difference of the deflection functions, $w_{1}(x)$ and $w_{2}(x)$, at the given cross-section:

$p(x)=K\left[w_{2}(x)-w_{1}(x)\right]$.

Here $K$ is the interfacial through-thickness spring constant of the assembly. In an approximate analysis, one can put

$K=\frac{1}{\frac{1-v_{1}}{3 E_{1}} h_{1}+\frac{1-v_{2}}{3 E_{2}} h_{2}+\frac{1-v_{0}}{E_{0}} h_{0}}$.

The first two terms in the denominator in this formula are the through-thickness compliances of the adherends, and the third term is the compliance of the bonding layer. The formula (17) is based on the following simple reasoning (assumptions):

1. Hooke's law can be applied to evaluate the throughthickness compliances;

2. by analogy with the longitudinal interfacial compliances, one can assume that the adherends are three times less compliant (for the same thickness) than the bonding layer; this is due to the fact that the entire thickness of the (relatively thin) bonding layer experiences peeling stresses, while only the inner portions, i.e., the portions adjacent to the interface, of the (relatively thick) adherends are subjected to appreciable peeling stresses, while their outer portions are understressed; and

3. the interfacial through-thickness compliance in the given cross-section, as well as the peeling stress in this cross-section, is not affected by the states of stress and strain in the adjacent cross-sections (also by analogy with the longitudinal interfacial compliances).

The relationship (16) reflects an assumption that it is the difference in the deflections $w_{1}(x)$ and $w_{2}(x)$ that determines the level of the peeling stress at the given cross-section. No peeling stress could possibly occur, if these displacements are the same.
By differentiation we obtain:

$p^{\prime \prime}(x)=K\left[w_{2}^{\prime \prime}(x)-w_{1}^{\prime \prime}(x)\right]$.

Solving the first equation in (14) for the curvature $w_{1}^{\prime \prime}(x)$ of the PCB, the second equation-for the curvature $w_{2}^{\prime \prime}(x)$ of the package and introducing the obtained expressions into the formula (18), the following equation for the peeling stress function, $p(x)$ can be obtained:

$p^{\prime \prime}(x)+4 \beta^{4} \int_{-a}^{x} \int_{-a}^{x} p(\xi) d \xi d \xi+\mu_{*} K T_{2}(x)$
$=\frac{K h_{1}}{2 D_{1}} \hat{T}+\frac{K}{D_{1}} \hat{M}$.

Here

$\beta=\sqrt[4]{\frac{K\left(D_{1}+D_{2}\right)}{4 D_{1} D_{2}}}$

is the parameter of the peeling stress and

$\mu_{*}=\frac{h_{1}}{2 D_{1}}-\frac{h_{2}}{2 D_{2}}$

is the parameter of the different flexural rigidities of the assembly components.

From (19) one obtains, by differentiation:

$p^{\prime \prime \prime}(x)+4 \beta^{4} \int_{-a}^{x} p(\xi) d \xi=-\mu_{*} K \tau(x)$

The next differentiation yields:

$p^{I V}(x)+4 \beta^{4} p(x)=-\mu_{*} K \tau^{\prime}(x)$.

The peeling stress $p(x)$ should be self-equilibrated with respect to the bending moments, as well as with respect to the lateral forces, so that the conditions of equilibrium

$\int_{-a}^{a} \int_{-a}^{x} p(\xi) d \xi d \xi=0, \quad \int_{-a}^{a} p(\xi) d \xi=0$.

should be fulfilled. These conditions can be translated, considering the Eqs. (19) and (22), into the following boundary conditions for the peeling stress function $p(x)$ :

$p^{\prime \prime}(a)=\frac{K \hat{M}}{D_{1}}+\frac{K h_{1} \hat{T}}{2 D_{1}}, \quad p^{\prime \prime \prime}(a)=-\mu_{*} K \tau(l)$

Note that the equation of the type (23) is encountered in the theory of beams lying on continuous elastic foundations (see e.g., [12, 13]), where, however, the role of the peeling stress function $p(x)$ is played by the deflection function $w(x)$. 


\subsection{Axial compliance of the assembly with consideration of its finite flexural rigidity}

Solving the first equation in (14) for the curvature $w_{1}^{\prime \prime}(x)$, the second equation-for the curvature $w_{2}^{\prime \prime}(x)$, differentiating the obtained expressions with respect to the coordinate $x$, and substituting the results into the Eq. (13), we obtain the following equation for the shearing stress function $\tau(x)$ :

$\kappa \tau^{\prime \prime}(x)-\lambda \tau(x)=\mu_{*} \int_{-a}^{x} p(\xi) d \xi$,

where

$\lambda=\lambda_{1}+\lambda_{2}+\frac{h_{1}^{2}}{4 D_{1}}+\frac{h_{2}^{2}}{4 D_{2}}=\lambda_{1}\left(4+3 v_{1}\right)+\lambda_{2}\left(4+3 v_{2}\right)$

is the total axial compliance of the assembly. This compliance is independent of the compliance of the bonding layer, as long as the bonding layer is thin and is comprised of a low-modulus material, i.e., as long as the axial compliance of the bonding layer is significantly greater than the compliance of the adherends. This is usually the case in the adhesively bonded or soldered assemblies employed in electronics packaging. As evident from the formula (27), the finite flexural rigidity of the assembly components results in a significantly greater total axial compliance of the assembly. This leads to somewhat higher shearing stresses compared to the situation when bending is small or is not considered.

\subsection{Higher order equations for the interfacial shearing and peeling stress functions}

From (26) one obtains by differentiation:

$\tau^{\prime \prime \prime}(x)-k^{2} \tau^{\prime}(x)=\frac{\mu_{*}}{\kappa} p(x)$

The next differentiation yields:

$\tau^{I V}(x)-k^{2} \tau^{\prime \prime}(x)=\frac{\mu_{*}}{\kappa} p^{\prime}(x)$,

where

$k=\sqrt{\frac{\lambda}{\kappa}}$

is the parameter of the interfacial shearing stress. This parameter is, in a way, similar to the parameter (20) of the interfacial peeling stress. Comparing the Eqs. (23) and (29), we conclude that the peeling stress affects the interfacial shearing stress in the same way as the shearing stress affects the peeling stress: one interfacial stress category depends on the gradient of the other with respect to the coordinate $x$. The functions, $p(x)$ and $\tau(x)$, become uncoupled when the parameter $\mu_{*}$ expressed by the Eq. (21) is next-to-zero and/or when the compliance of the bonding layer with respect to the corresponding stress is significant. Indeed, in this case the right parts of the Eqs. (23) and (29) are small, and these two equations become homogeneous and uncoupled.

The interfacial stress functions $\tau(x)$ and $p(x)$ obey, in effect, the same differential equation of the sixth order. Indeed, from (23) and (29) we obtain:

$\tau^{V I}(x)-k^{2} \tau^{I V}(x)+4 \beta^{4} \tau^{\prime \prime}(x)-\left(4 \beta^{4} k^{2}-\varsigma^{6}\right) \tau(x)=0$,

$p^{V I}(x)-k^{2} p^{I V}(x)+4 \beta^{4} p^{\prime \prime}(x)-\left(4 \beta^{4} k^{2}-\varsigma^{6}\right) p(x)=0$,

where

$\varsigma=\sqrt[6]{\frac{\mu_{*}^{2} K}{\kappa}}$

is the parameter of the assembly stiffness/compliance. This parameter increases with an increase in the through-thickness spring constant $K$ and with a decrease in its interfacial compliance $\kappa$, and is small, when the parameter $\mu$ of the flexural rigidities of the assembly components is small.

When the parameter (33) is zero, the Eqs. (31) and (32) yield:

$\Theta^{I V}(x)+4 \beta^{4} \Theta(x)=0$,

where the function $\Theta(x)$ is either

$\Theta(x)=\tau^{\prime \prime}(x)-\tau(x)$

or

$\Theta(x)=p^{\prime \prime}(x)-p(x)$,

depending on which interfacial stress is sought. The further analysis could be based on either the Eqs. (31) or (32), or on either the Eqs. (35) or (36), but with the appropriate and different boundary conditions.

\subsection{Boundary conditions}

From the Eq. (14), considering the formulas (7) and the conditions (24), one concludes that the deflection functions of the assembly components should satisfy the following boundary conditions:

$$
\begin{aligned}
& w_{1}^{\prime \prime}(a)=-\frac{\hat{M}}{D_{1}}-\frac{h_{1} \hat{T}}{2 D_{1}}, \quad w_{2}^{\prime \prime}(a)=0, \quad w_{1}^{\prime \prime \prime}(a)=\frac{h_{1}}{2 D_{1}} \tau(a), \\
& w_{2}^{\prime \prime \prime}(a)=-\frac{h_{2}}{2 D_{2}} \tau(a)
\end{aligned}
$$


Then the Eq. (12) results in the following conditions for the shearing stress function $\tau(x)$ :

$$
\begin{aligned}
\tau^{\prime}(a) & =-\frac{\lambda_{1}}{\kappa} \hat{T}-\frac{h_{1}}{2 \kappa D_{1}}\left(\hat{M}+\frac{h_{1}}{2} \hat{T}\right) \\
& =-\left(\lambda_{1}+\frac{h_{1}^{2}}{4 D_{1}}\right) \frac{\hat{T}}{\kappa}-\frac{h_{1} \hat{M}}{2 \kappa D_{1}}=-\eta \lambda_{1} \frac{\hat{T}}{\kappa} \\
\tau^{\prime \prime}(a) & -k^{2} \tau(a)=0,
\end{aligned}
$$

where

$$
\eta=1+\frac{h_{1}^{2}}{2 D_{1} \lambda_{1}}\left(1-2 \frac{\hat{M}}{h_{1} \hat{T}}\right)
$$

is the parameter of the boundary condition for the interfacial shearing stress. Note that the condition (39) follows also from the Eq. (26) and the second condition in (24). When bending is not considered $\left(D_{1} \rightarrow \infty\right)$, the condition (38) yields :

$\tau^{\prime}(a)=-\lambda_{1} \frac{\hat{T}}{\kappa}$.

Comparing the expressions (33) and (41), we conclude that the parameter (40) considers the effect of the assembly bending (finite flexural rigidity) on the interfacial shearing stress. In the absence of the external tensile forces $(\hat{T}=0)$ and when the effect of the shearing stress on the peeling stress is not accounted for $(\tau(x) \equiv 0)$, the conditions (25) yield:

$p^{\prime \prime}(a)=\frac{K \hat{M}}{D_{1}}, \quad p^{\prime \prime \prime}(a)=0$

\subsection{Simplified approach}

We use in this paper a simplified approach to evaluate the interfacial stresses. Namely, we assume that the shearing stress, $\tau(x)$, is not affected by the peeling stress, and can be found from the simplified equation

$\tau^{\prime \prime}(x)-k^{2} \tau(x)=0$.

This equation can be obtained from the Eq. (26) by simply putting its right part equal to zero and using the formula (30) for the parameter of the interfacial shearing stress. The acceptability of such an assumption has been demonstrated earlier for the thermally induced stresses by comparing the results of the simplified analytical solution with the finite element analysis (FEA) data [16] for a variety of the bonded assemblies. It is natural to assume that this assumption is applicable to the "mechanical" stresses as well. After the shearing stress, $\tau(x)$, is determined, the peeling stress, $p(x)$, can be evaluated from the Eq. (23).
The shearing stress function, $\tau(x)$, defined by the Eq. (43), satisfies the boundary condition (39). The solution to the Eq. (43) that satisfies also the condition (33) is as follows:

$\tau(x)=\frac{\tau^{\prime}(a)}{k} \frac{\sinh k x}{\cosh k a}=-\eta \lambda_{1} \frac{\hat{T}}{k \kappa} \frac{\sinh k x}{\cosh k a}$,

where the derivative $\tau^{\prime}(a)$ is expressed by the formula (38).

The maximum value of the interfacial shearing stress in the solder material takes place at the ends of the package:

$\tau(a)=-\eta \lambda_{1} \frac{\hat{T}}{k \kappa} \tanh k a$

For long enough packages with stiff interfaces $(k l \geq 2.5)$ the formula (45) can be simplified:

$\tau(a)=-\eta \lambda_{1} \frac{\hat{T}}{k \kappa}$

As to the peeling stress, the solution to the Eq. (23) for this stress can be sought in the form:

$p(x)=C_{0} \cosh \beta x \cos \beta x+C_{2} \sinh \beta x \sin \beta x-p_{0} \frac{\cosh k x}{\cosh k a}$.

where

$p_{0}=\frac{\mu_{*} K \tau^{\prime}(a)}{k^{4}+4 \beta^{4}}=-\eta \lambda_{1} \frac{\hat{T}}{\kappa} \frac{\mu_{*} K}{k^{4}+4 \beta^{4}}$.

The first two terms in the solution (47) provide the general solution to the homogeneous equation that corresponds to the Eq. (23), i.e., an equation that can be obtained by putting to zero the right part of the Eq. (23). The third term is the particular solution to the inhomogeneous Eq. (23).

Introducing the solution (47) into the conditions (25), the following algebraic equations for the constants $C_{0}$ and $C_{2}$ of integration can be obtained:

$$
\left.\begin{array}{l}
-C_{0} \sinh \beta a \sin \beta a+C_{2} \cosh \beta a \cos \beta a=p_{1} \\
-C_{0}(\cosh \beta a \sin \beta a+\sinh \beta a \cos \beta a)-C_{2}(\cosh \beta a \sin \beta a \\
-\sinh \beta a \cos \beta a)=p_{2}
\end{array}\right\}
$$

where the notations

$$
\left.\begin{array}{l}
p_{1}=\frac{k^{2} p_{0}+p^{\prime \prime}(a)}{2 \beta^{2}}=\frac{2 \beta^{2} \hat{M}}{1+\frac{D_{1}}{D_{2}}}\left[1+\frac{h_{1} \hat{T}}{2 \hat{M}}\left(1-\frac{2 D_{1}}{h_{1}} \mu_{*} \eta \frac{\frac{\lambda_{1}}{\lambda}}{1+4 \frac{\beta^{4}}{k^{4}}}\right)\right] \\
p_{2}=\frac{k^{3} p_{0} \tanh k a+p^{\prime \prime \prime}(a)}{2 \beta^{3}}=2 \beta k \hat{T} \mu_{*} \eta \frac{\frac{\lambda_{1}}{\lambda}}{1+\frac{k^{4}}{4 \beta^{4}}} \frac{D_{1} D_{2}}{D_{1}+D_{2}}
\end{array}\right\}
$$

are used.

The Eq. (49) yield: 
$\left.\begin{array}{l}C_{0}=2 \frac{p_{1}(\sinh \beta a \cos \beta a-\cosh \beta a \sin \beta a)-p_{2} \cosh \beta a \cos \beta a}{\sinh 2 \beta a+\sin 2 \beta a} \\ C_{2}=2 \frac{p_{1}(\cosh \beta a \sin \beta a+\sinh \beta a \cos \beta a)-p_{2} \sinh \beta a \sin \beta a}{\sinh 2 \beta a+\sin 2 \beta a}\end{array}\right\}$,

and the solution (47) leads to the following expression for the interfacial peeling stress:

$$
\begin{aligned}
p(x)= & \frac{2}{\sinh 2 \beta a+\sin 2 \beta a}\left[p_{1}((\sinh \beta a \cos \beta a\right. \\
& -\cosh \beta a \sin \beta a) \cosh \beta x \cos \beta x+ \\
& +(\sinh \beta a \cos \beta a+\cosh \beta a \sin \beta a) \sinh \beta x \sin \beta x) \\
& -p_{2}(\cosh \beta a \cos \beta a \cosh \beta x \cos \beta x+ \\
& +\sinh \beta a \sin \beta a \sinh \beta x \sin \beta x)]-p_{0} \frac{\cosh k x}{\cosh k a} .
\end{aligned}
$$

expression (54) can be written in the following simple form:

$p(x)=e^{-\beta x}\left[\left(p_{1}-p_{2}\right) \cos \beta x-p_{1} \sin \beta x\right]-p_{0} e^{-k x}$.

\section{Numerical example}

In the numerical examples below the results of the Ref. [15] are used to calculate the forces and moments (1) that the $\mathrm{PCB} /$ package assembly experiences as the consequence of the drop impact load applied to the nondeformable contour of the PCB.

\subsection{Input data}

\begin{tabular}{llll}
\hline Structural element & PCB & Package & BGA \\
\hline Element \# & 1 & 2 & 0 \\
Young's Modulus, $E, \mathrm{~kg} / \mathrm{mm}^{2}$ & 2321.4 & 8775.5 & 5510.0 \\
Poisson's ratio, $v$ & 0.40 & 0.25 & 0.35 \\
Thickness/height, $h$, & 1.5 & 2.0 & 0.6 \\
Shear modulus, $G, \mathrm{~kg} / \mathrm{mm}^{2}$ & 892.7 & 3387.3 & 2040.7 \\
Axial compliance, $\lambda, \mathrm{mm} / \mathrm{kg}$ & $172.3098 \times 10^{-6}$ & $42.7326 \times 10^{-6}$ & $\times$ \\
Interfacial compliance, $\kappa, \mathrm{mm}^{3} / \mathrm{kg}$ & $560.0986 \times 10^{-6}$ & $196.8136 \times 10^{-6}$ & $245.0140 \times 10^{-6}$ \\
Flexural rigidity, $D, \mathrm{~kg} \mathrm{~mm}$ & 777.2545 & 6240.3556 & $\times$ \\
Distributed mass, $m, \mathrm{~kg} \mathrm{~s} / \mathrm{mm}^{3}$ & $7.8780 \times 10^{-10}$ & $4.5306 \times 10^{-6}$ & $16.6120 \times 10^{-6}$ \\
Size (half-length), $\mathrm{mm}$ & $a=150$ & $l=20$ & $\times$ \\
Drop height $H=1.273 \mathrm{~m}$; Initial velocity & $V_{0}=\sqrt{2 g H}=4996 \mathrm{~mm} / \mathrm{s} ;$ & \\
\hline
\end{tabular}

The maximum value of the peeling stress takes place at the assembly end:

$p_{\text {max }}=p(a)=p_{1} \frac{\sinh 2 \beta a-\sin 2 \beta a}{\sinh 2 \beta a+\sin 2 \beta a}-p_{2}-p_{0}$.

In the case of a sufficiently long assembly (large $a$ values) and/or an assembly with a stiff enough bonding layer (large $\beta$ values), the solution (52) can be simplified:

$$
\begin{aligned}
p(x)= & e^{-\beta(a-x)}\left[\left(p_{1}-p_{2}\right) \cos [\beta(a-x)]-p_{1} \sin [\beta(a-x)]\right] \\
& -p_{0} e^{-k(a-x)},
\end{aligned}
$$

and the maximum peeling stress is

$p_{\max }=p(a)=p_{1}-p_{2}-p_{0}$

If one puts the origin of the coordinate $x$ at the assembly end and directs this coordinate inwards the assembly, the

\subsection{Computed data}

Distributed mass of the "heavy" PCB

- with BGA solder $m=7.8780 \times 10^{-10}+4.5306 \times$ $10^{-10}=12.4086 \times 10^{-10} \mathrm{~kg} \mathrm{~s}^{2} / \mathrm{mm}^{3}$

- with CGA solder $m=7.8780 \times 10^{10}+16.6120 \times$ $10^{-10}=24.4900 \times 10^{-10} \mathrm{~kg} \mathrm{~s}^{2} / \mathrm{mm}^{3}$

Actual mass of the PCB

- with BGA solder $M_{a}=4 m a^{2}=4 \times 12.4086 \times$ $10^{-10} \times 150^{2}=111.6774 \times 10^{-6} \mathrm{~kg} \mathrm{~s}^{2} / \mathrm{mm}$

- with CGA solder $M_{a}=4 m a^{2}=4 \times 24.4900 \times$ $10^{-10} \times 150^{2}=220.4100 \times 10^{-6} \mathrm{~kg} \mathrm{~s}^{2} / \mathrm{mm}$

Generalized mass of the PCB

- with BGA solder $M=m a^{2}=12.4086 \times 10^{-10} \times$ $150^{2}=27.9193 \times 10^{-6} \mathrm{~kg} \mathrm{~s}^{2} / \mathrm{mm}$ 
- with CGA solder $M=m a^{2}=24.4900 \times 10^{-10} \times$ $150^{2}=55.1025 \times 10^{-6} \mathrm{~kg} \mathrm{~s}^{2} / \mathrm{mm}$

Linear frequency of the impact induced PCB vibrations

- with BGA solder $\omega=\frac{\pi^{2}}{2 a} \sqrt{\frac{D}{M}}=\frac{\pi^{2}}{300} \sqrt{\frac{777.2545}{27.9193 \times 10^{-6}}}=$ $173.5830 \mathrm{~s}^{-1}$

- with CGA solder $\omega=\frac{\pi^{2}}{2 a} \sqrt{\frac{D}{M}}=\frac{\pi^{2}}{300} \sqrt{\frac{777.2545}{55.1025 \times 10^{-6}}}=$ $123.5588 \mathrm{~s}^{-1}$

Linear amplitude of the impact induced PCB vibrations

- $\quad$ with BGA solder $A_{0}=\frac{16}{\pi^{2}} \frac{V_{0}}{\omega}=\frac{16}{\pi^{2}} \frac{4996}{173.5830}=46.66 \mathrm{~mm}$

- $\quad$ with CGA solder $A_{0}=\frac{16}{\pi^{2}} \frac{V_{0}}{\omega}=\frac{16}{\pi^{2}} \frac{4996}{123.5588}=65.55 \mathrm{~mm}$

Maximum linear acceleration (deceleration) of the PCB

- with BGA solder $\ddot{f}_{\max }=-\omega^{2} A_{0}=173.5830^{2} \times$ $46.66=-1405915.1611 \mathrm{~mm} / \mathrm{s}^{2}=-143.5 \mathrm{~g}$

- with CGA solder $\ddot{f}_{\max }=-\omega^{2} A_{0}=123.5588^{2} \times$ $65.55=-1000737.2361 \mathrm{~mm} / \mathrm{s}^{2}=-102.1 \mathrm{~g}$

Parameter of nonlinearity of the PCB impact induced vibrations

- with BGA solder $\mu=\frac{3 \pi^{4}}{32}(3-v)(1+v) \frac{D}{M a^{2} h_{1}^{2}}=\frac{3 \pi^{4}}{32} \times 2.6 \times$ $1.4 \times \frac{777.2545}{27.9193 \times 10^{-6} \times 150^{2} \times 1.5^{2}}=18,279.5023 \mathrm{~mm}^{-2} / \mathrm{s}^{2}$

- with CGA solder $\mu=\frac{3 \pi^{4}}{32}(3-v)(1+v) \frac{D}{M a^{2} h_{1}^{2}}=\frac{3 \pi^{4}}{32} \times$ $2.6 \times 1.4 \times \frac{777.2545}{55.1025 \times 10^{-6} \times 150^{2} \times 1.5^{2}}=9261.8467 \mathrm{~mm}^{-2} / \mathrm{s}^{2}$

Dimensionless parameter of nonlinearity

- $\quad$ with BGA solder $\bar{\mu}=\mu\left(\frac{A_{0}}{\omega}\right)^{2}=18,279.5023\left(\frac{46.66}{173.5830}\right)^{2}=$ 1320.8073

- with CGA solder $\bar{\mu}=\mu\left(\frac{A_{0}}{\omega}\right)^{2}=9261.8467\left(\frac{65.55}{123.5588}\right)^{2}=$ 2606.7274

Factor of the nonlinear amplitude (ratio of the nonlinear amplitude to the linear amplitude)

- with BGA solder $\eta_{A}=\sqrt{\frac{\sqrt{1+2 \bar{\mu}}-1}{\bar{\mu}}}=$ $\sqrt{\frac{\sqrt{1+2 \times 1320.8973}-1}{1320.8073}}=0.1954$

- with CGA solder $\sqrt{\frac{\sqrt{1+2 \times 2606.7274}-1}{2606.7274}}=0.1653$

Nonlinear amplitude

- with BGA solder $A=\eta_{A} A_{0}=0.1954 \times 46.66=$ $9.1174 \mathrm{~mm}$
- with CGA solder $A=\eta_{A} A_{0}=0.1653 \times 65.55=$ $10.8354 \mathrm{~mm}$

Note that while the (hypothetical) linear amplitude of the dynamic response of the "heavy" PCB with the CGA solder is by the factor of 1.40 greater than in the case when CGA system is used, this factor is only 1.19 for the nonlinear response.

Parameter of the nonlinear frequency is

- with BGA solder $\sigma=\sqrt{\omega^{2}+\mu A^{2}}=$ $\sqrt{173.5830^{2}+18,279.5023 \times 9.1174^{2}}=1244.85 \mathrm{~s}^{-1}$

- with $\mathrm{CGA} \quad$ solder $\quad \sigma=\sqrt{\omega^{2}+\mu A^{2}}=$ $\sqrt{123.5588^{2}+9261.8467 \times 10.8354^{2}}=1050.07 \mathrm{~s}^{-1}$

Maximum nonlinear acceleration (deceleration) is

- with BGA solder $\ddot{f}_{\max }=-\sigma^{2} A=1244.85^{2} \times$ $9.1174=-14,128,792.7912 \mathrm{~mm} / \mathrm{s}^{2}=-1441.71 \mathrm{~g}$

- with BGA solder $\ddot{f}_{\text {max }}=-\sigma^{2} A=1057.07^{2} \times$ $10.8354=-12,107,443.2902 \mathrm{~mm} / \mathrm{s}^{2}=-1235.45 \mathrm{~g}$

Modulus of the elliptic function

- with BGA solder $k=\frac{A}{\sigma} \sqrt{\frac{\mu}{2}}=\frac{9.1174}{1244.85} \sqrt{\frac{18,279.5023}{2}}=$ 0.7002

- with CGA solder $k=\frac{A}{\sigma} \sqrt{\frac{\mu}{2}}=\frac{10.8354}{1050.07} \sqrt{\frac{9261.8467}{2}}=$ 0.7022

This modulus turned out to be approximately the same for the BGA and CGA cases.

Elliptic integral is $K(k) \approx 1.847$.

The nonlinear frequency is

$p=\frac{\pi \sigma}{2 K(k)}=\frac{\pi}{2} \frac{1244.85}{1.847}=1058.69 \mathrm{~s}^{-1}$

- with BGA solder $p=\frac{\pi \sigma}{2 K(k)}=\frac{\pi}{2} \frac{1057.07}{1.847}=898.99 \mathrm{~s}^{-1}$

- with CGA solder.

The nonlinear frequency exceeds dramatically the linear frequency. The nonlinear frequency of the $\mathrm{PCB}$ vibrations is greater by the factor of 1.18 for the case of the BGA solder in comparison with the case of the CGA solder. This factor was 1.40 in the linear case.

Tensile force (per unit assembly width) in the midportion of the PCB is

- with BGA solder $\hat{T}=\frac{E_{1}}{1-v_{1}} \frac{2-v_{1}}{2} h_{1}\left(\frac{\pi A}{4 a}\right)^{2}=\frac{2321.4}{0.6} \frac{1.6}{2}$ $1.5\left(\frac{\pi \times 9.1174}{4 \times 150}\right)^{2}=10.5808 \mathrm{~kg} / \mathrm{mm}$ 
- with CGA solder $\hat{T}=\frac{E_{1}}{1-v_{1}} \frac{2-v_{1}}{2} h_{1}\left(\frac{\pi A}{4 a}\right)^{2}=\frac{2321.4}{0.6} \frac{1.6}{2}$ $1.5\left(\frac{\pi \times 10.8354}{4 \times 150}\right)^{2}=14.9440 \mathrm{~kg} / \mathrm{mm}$

Bending moment (per unit assembly width) in the midportion of the PCB is $\hat{M}=\frac{E_{1}}{1-v_{1}} \frac{h_{1}^{3}}{12} A\left(\frac{\pi}{2 a}\right)^{2}=\frac{2321.4}{0.6} \frac{1.5^{3}}{12} \times$ $9.1174\left(\frac{\pi}{2 \times 150}\right)^{2}=1.0880 \mathrm{~kg}$

- with BGA solder and $\hat{M}=\frac{E_{1}}{1-v_{1}} \frac{h_{1}^{3}}{12} A\left(\frac{\pi}{2 a}\right)^{2}=$ $\frac{2321.4}{0.6} \frac{1.5^{3}}{12} \times 10.8354\left(\frac{\pi}{2 \times 150}\right)^{2}=1.2930 \mathrm{~kg}$

- with CGA solder

Thus, both the tensile force and the bending moment applied to the PCB in the proximity of the package location are higher when the CGA design is employed.

Axial compliances are

$\lambda_{1}=\frac{1-v_{1}}{E_{1} h_{1}}=\frac{0.6}{2321.4 \times 1.5}=172.3098 \times 10^{-6} \mathrm{~mm} / \mathrm{kg}$

for the PCB

$\lambda_{2}=\frac{1-v_{2}}{E_{2} h_{2}}=\frac{0.75}{8775.5 \times 2.0}=42.7326 \times 10^{-6} \mathrm{~mm} / \mathrm{kg}$

and for the package.

The total axial compliance of the $\mathrm{PCB} /$ package assembly with consideration of the finite compliance of the PCB and the package is

$$
\begin{aligned}
\lambda= & \lambda_{1}\left(4+3 v_{1}\right)+\lambda_{2}\left(4+3 v_{2}\right)=172.3098 \times 10^{-6} \\
& \times 5.2+42.7326 \times 10^{-6} \times 4.75 \\
= & 1098.9909 \times 10^{-6} \mathrm{~mm} / \mathrm{kg}
\end{aligned}
$$

Interfacial compliances for the PCB and the package are $\kappa_{1}=\frac{h_{1}}{3 G_{1}}=\frac{1.5}{3 \times 892.7}=560.0986 \times 10^{-6} \mathrm{~mm}^{3} / \mathrm{kg}$

and

$\kappa_{2}=\frac{h_{2}}{3 G_{2}}=\frac{2.0}{3 \times 3387.3}=196.8136 \times 10^{-6} \mathrm{~mm}^{3} / \mathrm{kg}$

The interfacial compliance of the BGA solder is

$\kappa_{0}=\frac{h_{0}}{G_{0}}=\frac{0.5}{2040.7}=245.0140 \times 10^{-6} \mathrm{~mm}^{3} / \mathrm{kg}$

The interfacial compliance of the CGA system is

$\kappa_{0}=\frac{h_{0}}{G_{0}}=\frac{2.2}{2040.7}=1078.0614 \times 10^{-6} \mathrm{~mm}^{3} / \mathrm{kg}$

The total interfacial compliance of the $\mathrm{PCB} /$ package assembly is

$$
\begin{aligned}
\kappa= & \kappa_{0}+\kappa_{1}+\kappa_{2} \\
= & 245.0140 \times 10^{-6}+560.0986 \times 10^{-6}+196.8136 \\
& \times 10^{-6} \\
= & 1001.9262 \times 10^{-6} \mathrm{~mm}^{3} / \mathrm{kg}
\end{aligned}
$$

in the case when the BGA solder is used and is

$$
\begin{aligned}
\kappa= & \kappa_{0}+\kappa_{1}+\kappa_{2} \\
= & 1078.0614 \times 10^{-6}+560.0986 \times 10^{-6}+196.8136 \\
& \times 10^{-6} \\
= & 1834.9732 \times 10^{-6} \mathrm{~mm}^{3} / \mathrm{kg}
\end{aligned}
$$

in the case of the CGA solder system.

Parameter of the interfacial shearing stress is

$k=\sqrt{\frac{\lambda}{\kappa}}=\sqrt{\frac{1098.9909 \times 10^{-6}}{1001.9262 \times 10^{-6}}}=1.0473 \mathrm{~mm}^{-1}$

in the case of the BGA solder and is

$k=\sqrt{\frac{\lambda}{\kappa}}=\sqrt{\frac{1098.9909 \times 10^{-6}}{1834.9732 \times 10^{-6}}}=0.7739 \mathrm{~mm}^{-1}$

in the case of the CGA solder.

Parameter of the boundary condition for the interfacial shearing stress is

$$
\begin{aligned}
\eta= & 1+\frac{h_{1}^{2}}{2 D_{1} \lambda_{1}}\left(1-2 \frac{\hat{M}}{h_{1} \hat{T}}\right) \\
= & 1+\frac{2.25}{2 \times 777.2545 \times 172.3098 \times 10^{-6}} \\
& \left(1-2 \frac{1.088}{1.5 \times 10.5808}\right)=8.2483
\end{aligned}
$$

- with BGA solder $\eta=1+\frac{h_{1}^{2}}{2 D_{1} \lambda_{1}}\left(1-2 \frac{\hat{M}}{h_{1} \hat{T}}\right)=1+$ $\frac{2.25}{2 \times 777.2545 \times 172.3098 \times 10^{-6}}\left(1-2 \frac{1.2930}{1.5 \times 14.9440}\right)=8.4309$

- with CGA solder.

Maximum interfacial shearing stress is

$$
\begin{aligned}
\tau_{\max }= & -\eta \lambda_{1} \frac{\hat{T}}{\mathrm{k \kappa}} \\
= & -8.2483 \times 172.3098 \\
& \times 10^{-6} \frac{10.5808}{1.0473 \times 1001.9262 \times 10^{-6}} \\
= & -14.3313 \mathrm{~kg} / \mathrm{mm}^{2}
\end{aligned}
$$

in the case of the BGA solder and is

$$
\begin{aligned}
\tau_{\max }= & -\eta \lambda_{1} \frac{\hat{T}}{k \kappa} \\
= & -8.4309 \times 172.3098 \\
& \times 10^{-6} \frac{14.9440}{0.7739 \times 1834.9732 \times 10^{-6}} \\
= & -15.2875 \mathrm{~kg} / \mathrm{mm}^{2}
\end{aligned}
$$


in the case of the CGA solder.

Thus, based on the carried out example, the application of the CGA instead of the BGA did not practically make a difference in the level of the maximum dynamic interfacial shearing stress. The unfavorable effect of the elevated weight (mass) of the CGA system outweighed the favorable effect of its interfacial compliance.

Let us take a look now on whether the application of the CGA instead of BGA leads to an appreciable difference for the maximum peeling stress.

The interfacial through-thickness spring constant is

$$
\begin{aligned}
K & =\frac{1}{\frac{1-v_{1}}{3 E_{1}} h_{1}+\frac{1-v_{2}}{3 E_{2}} h_{2}+\frac{1-v_{0}}{E_{0}} h_{0}} \\
& =\frac{0.6}{3 \times 2321.4} 1.5+\frac{0.75}{3 \times 8775.5} 2.0+\frac{0.65}{5510.0} 0.6 \\
& =3891.2081 \mathrm{~kg} / \mathrm{mm}
\end{aligned}
$$

with the BGA solder and

$$
\begin{aligned}
K & =\frac{1}{\frac{1-v_{1}}{3 E_{1}} h_{1}+\frac{1-v_{2}}{3 E_{2}} h_{2}+\frac{1-v_{0}}{E_{0}} h_{0}} \\
& =\frac{0.6}{\frac{0.6}{3 \times 2321.4} 1.5+\frac{0.75}{3 \times 8775.5} 2.0+\frac{0.65}{5510.0} 2.2} \\
& =2243.4737 \mathrm{~mm}^{3} / \mathrm{kg}
\end{aligned}
$$

with the CGA solder.

The parameter of the peeling stress is then

$$
\begin{aligned}
\beta & =\sqrt[4]{\frac{K\left(D_{1}+D_{2}\right)}{4 D_{1} D_{2}}} \\
& =\sqrt[4]{\frac{3891.2081(777.2545+6240.3556)}{4 \times 777.2545 \times 6240.3556}} \\
& =1.0892 \mathrm{~mm}^{-1}
\end{aligned}
$$

in the case of the BGA system and

$$
\begin{aligned}
\beta & =\sqrt[4]{\frac{K\left(D_{1}+D_{2}\right)}{4 D_{1} D_{2}}} \\
& =\sqrt[4]{\frac{2243.4737(777.2545+6240.3556)}{4 \times 777.2545 \times 6240.3556}} \\
& =0.9491 \mathrm{~mm}^{-1}
\end{aligned}
$$

in the case of the CGA design.

The parameter of the different flexural rigidities of the assembly components is

$$
\begin{aligned}
\mu_{*} & =\frac{h_{1}}{2 D_{1}}-\frac{h_{2}}{2 D_{2}}=\frac{1.5}{2 \times 777.2545}-\frac{2.0}{2 \times 6240.3556} \\
& =804.6876 \times 10^{-6} \mathrm{~kg}^{-1}
\end{aligned}
$$

The parameter $p_{1}$ of the peeling stress is

$$
\begin{aligned}
p_{1}= & \frac{2 \beta^{2} \hat{M}}{1+\frac{D_{1}}{D_{2}}}\left[1+\frac{h_{1} \hat{T}}{2 \hat{M}}\left(1-\frac{2 D_{1}}{h_{1}} \mu_{*} \eta \frac{\frac{\lambda_{1}}{\lambda}}{1+4 \frac{\beta^{4}}{k^{4}}}\right)\right] \\
= & \frac{2 \times 1.0892^{2} \times 1.088}{1.1246}\left[1+\frac{1.5 \times 10.5808}{2 \times 1.088}\right. \\
& \left(1-\frac{2 \times 777.2545}{1.5} \times 804.6876 \times 10^{-6}\right. \\
& \left.\left.\times 8.2483 \times \frac{0.1568}{5.6796}\right)\right]=15.8590 \mathrm{~kg} / \mathrm{mm}^{2}
\end{aligned}
$$

in the case of the BGA and is

$$
\begin{aligned}
p_{1}= & \frac{2 \beta^{2} \hat{M}}{1+\frac{D_{1}}{D_{2}}}\left[1+\frac{h_{1} \hat{T}}{2 \hat{M}}\left(1-\frac{2 D_{1}}{h_{1}} \mu_{*} \eta \frac{\frac{\lambda_{1}}{\lambda}}{1+4 \frac{\beta^{4}}{k^{4}}}\right)\right] \\
= & \frac{2 \times 0.9491^{2} \times 1.2930}{1.1246}\left[1+\frac{1.5 \times 14.9440}{2 \times 1.2930}\right. \\
& \left(1-\frac{2 \times 777.2545}{1.5} \times 804.6876 \times 10^{-6}\right. \\
& \left.\left.\times 8.4309 \times \frac{0.1568}{10.0483}\right)\right]=18.0564 \mathrm{~kg} / \mathrm{mm}^{2}
\end{aligned}
$$

in the case of CGA.

The parameter $p_{2}$ is as small as

$$
\begin{aligned}
p_{2}= & 2 \beta k \hat{T} \mu_{*} \eta \frac{\frac{\lambda_{1}}{\lambda}}{1+\frac{k^{4}}{4 \beta^{4}}} \frac{D_{1} D_{2}}{D_{1}+D_{2}}=2 \times 1.0892 \times 1.0473 \\
& \times 10.5808 \times 804.6876 \times 10^{-6} \times 8.2483 \times \frac{0.1568}{5.6796} \\
= & 0.0044 \mathrm{~kg} / \mathrm{mm}^{2}
\end{aligned}
$$

in the case of BGA and

$$
\begin{aligned}
p_{2}= & 2 \beta k \hat{T} \mu_{*} \eta \frac{\frac{\lambda_{1}}{\lambda}}{1+\frac{k^{4}}{4 \beta^{4}}} \frac{D_{1} D_{2}}{D_{1}+D_{2}}=2 \times 0.9491 \times 0.7739 \\
& \times 14.9440 \times 804.6876 \times 10^{-6} \times 8.4309 \times \frac{0.1568}{10.0483} \\
= & 0.0023 \mathrm{~kg} / \mathrm{mm}^{2}
\end{aligned}
$$

in the case of CGA.

The parameter $p_{0}$ is

$$
\begin{aligned}
p_{0}= & -\eta \lambda_{1} \frac{\hat{T}}{\kappa k^{4}} \frac{\mu_{*} K}{1+4 \frac{\beta^{4}}{k^{4}}}=-8.2483 \times 172.3098 \\
& \times 10^{-6} \frac{10.5808}{1001.9262 \times 10^{-6} \times 1.0473^{4}} \\
& \times \frac{804.6876 \times 10^{-6} \times 3891.2081}{5.6796}=-6.8781 \mathrm{~kg} / \mathrm{mm}^{2}
\end{aligned}
$$

in the case of BGA and 


$$
\begin{aligned}
p_{0}= & -\eta \lambda_{1} \frac{\hat{T}}{\kappa k^{4}} \frac{\mu_{*} K}{1+4 \frac{\beta^{4}}{k^{4}}}=-8.4309 \times 172.3098 \\
& \times 10^{-6} \frac{14.9440}{1834.9732 \times 10^{-6} \times 0.7739^{4}} \\
& \times \frac{804.6876 \times 10^{-6} \times 2243.4737}{10.0483}=-5.9257 \mathrm{~kg} / \mathrm{mm}^{2}
\end{aligned}
$$

in the case of CGA.

The maximum peeling stress is

$$
\begin{aligned}
p_{\max } & =p_{1}-p_{2}-p_{0}=15.8590-0.0044+6.8781 \\
& =22.7327 \mathrm{~kg} / \mathrm{mm}^{2}
\end{aligned}
$$

in the case of BGA and

$$
\begin{aligned}
p_{\max } & =p_{1}-p_{2}-p_{0}=18.0564-0.0023+5.9257 \\
& =23.9798 \mathrm{~kg} / \mathrm{mm}^{2}
\end{aligned}
$$

in the case of BGA.

The calculated stresses are summarized in the table:

\begin{tabular}{lll}
\hline Max. stresses, $\mathrm{kg} / \mathrm{mm}^{2}$ & Shearing & Peeling \\
\hline System & & \\
BGA & 14.331 & 22.733 \\
CGA & 15.285 & 23.980 \\
\hline
\end{tabular}

These (dynamic) stresses are significantly higher, by an order of magnitude, than the predicted thermal stresses. The calculated dynamic shearing stress is by about $6.7 \%$ higher, and the predicted peeling stress is by about $5.5 \%$ higher, when the CGA technology is used, as compared to the BGA design.

\section{Conclusions}

- The numerical example carried out for a rather arbitrary, but realistic, input data has indicated that the impact induced stresses in solder joints can exceed significantly the thermally induced stresses and that indeed the dynamic stresses in the CGA are even slightly greater than in the BGA interconnections. This means particularly that the physically meaningful drop height in accelerated and qualification tests should be carefully selected and that this height should be different, when BGA and CGA interconnections are considered.

- The following future work is important and is currently considered:
1. Computerize the obtained analytical relationships for the thermally induced and dynamic shearing and peeling stresses, and conduct serial computations to evaluate the roles of different factors affecting the induced stresses in thermal and dynamic tests;

2. Carry out FEA computations to verify the accuracy of the analytical modeling;

3. Design and build the experimental vehicles for thermal and dynamic testing of BGA and CGA interconnections; the developed analytical models can be quite helpful in doing that.

\section{References}

1. E. Suhir, Stresses in bi-metal thermostats. ASME J. Appl. Mech. 53(3), 657-660 (1986)

2. E. Suhir, Calculated thermally induced stresses in adhesively bonded and soldered assemblies, in Proceedings of the International Symposium on Microelectronics, ISHM, 1986, Atlanta, Georgia (1986)

3. E. Suhir, Interfacial stresses in bi-metal thermostats. ASME J. Appl. Mech. 56(3), 595-600 (1989)

4. E. Suhir, Thermal stress failures in microelectronics and photonics: prediction and prevention. Future Circuits Int. 5, 83-89 (1999)

5. Z. Kovac et al., Compliant Interface for Semiconductor Chip and Method Therefor, US Patent \#6,133,639 (2000)

6. T.H. DiStefano et al., Compliant Microelectronic Mounting Device, US Patent \#6,370,032 (2002)

7. Z. Kovac et al., Methods for Making Electronic Assemblies Including Compliant Interfaces, US Patent \#6,525,429 (2003)

8. E. Suhir, Thermal stress in an adhesively bonded joint with a low modulus adhesive layer at the ends. Appl. Phys. J. (2003)

9. E. Paterson et al., Mechanical Highly Compliant Thermal Interface Pad, US Patent \#6,910,271 (2005)

10. Z. Kovac et al., Methods of Making Microelectronic Assemblies Including Compliant Interfaces, US Patent \#6,870,272 (2005)

11. E. Suhir, M. Vujosevic, Interfacial stresses in a bi-material assembly with a compliant bonding layer. J. Phys. D: Appl. Phys. 41(11), 115504 (2008). doi:10.1088/0022-3727/41/11/115504

12. S.P. Timoshenko, Strength of Materials, 3rd edn. (Van Nostrand Company Inc, Princeton, NJ, 1955)

13. E. Suhir, Structural Analysis in Microelectronic and Fiber Optic Systems (Van-Nostrand, New York, 1991)

14. E. Suhir, R. Ghaffarian, J. Nicolics, Could thermal stresses in a BGA/CGA-system be evaluated from a model intended for a homogeneously bonded assembly? J. Mater. Sci.: Mater. Electron. 27(1), 570-579 (2016). doi:10.1007/s10854-015-3790-9

15. E. Suhir, R. Ghaffarian, Board level drop test: exact solution to the problem of the nonlinear dynamic response of a PCB to the drop impact. J. Mater. Sci.: Mater. Electron. (2016). doi:10.1007/ s10854-016-4988-1

16. V. Mishkevich, E. Suhir, Simplified approach to the evaluation of thermally induced stresses in bi-material structures, in Structural Analysis in Microelectronics and Fiber Optics, ed. by E. Suhir (ASME Press, New York, 1993) 